\title{
Comparison of Cone-beam Computed Tomography and Periapical Radiography to Determine the Proximity of Periapical Lesions to Anatomical Structures in Premaxillary Area prior to Surgical Endodontics: A Clinical Study
}

\author{
Kesha Sheth ${ }^{1}$, Sonali Kapoor², Shilpi Daveshwar ${ }^{3}$
}

\begin{abstract}
Aim: In the anterior maxillary region when periapical (PA) surgery is contemplated, knowledge of relation between root apices and lesion, spatial relationship of tooth root to adjacent anatomical structures is essential. The aim of this study was to compare cone-beam computed tomography $(\mathrm{CBCT})$ and $\mathrm{PA}$ radiography to determine the proximity of PA lesions to anatomical structures in the premaxillary area for decisionmaking before apical surgery.

Materials and methods: Twenty subjects were selected. Three endodontists viewed PA radiographs and CBCT images. These observers (1) determined proximity of lesion to anatomical structures and (2) made decisions on treatment based on each imaging modality. Chi-square test was used to check the relationship between the treatment plans given by PA radiographs and CBCT images. Reliability of the observations was evaluated using intraclass correlation (ICC).

Results: Significant difference was noted in the treatment plan selected by the observers using the two modalities $(p<0.05)$.

Conclusion: After the lesions were observed with $\mathrm{CBCT}$, there was an increase in the number of teeth involved with the lesion. A change in the observer's treatment plan was recorded after viewing the CBCT scans.

Keywords: Anterior maxilla, Cone-beam computed tomography, Periapical lesion, Periapical radiography, Periapical surgery. International Journal of Clinical Pediatric Dentistry (2020): 10.5005/jp-journals-10005-1783
\end{abstract}

\section{INTRODUCTION}

Complete oral examination and high-resolution imaging are crucial diagnostic tools for treatment planning when periapical (PA) surgery is contemplated. ' Clinical diagnosis is supported by accurate imaging and helps in determining the number of roots in the involved tooth, the number of affected roots in the involved tooth, the distance between the root apices, the extent of the PA lesion, and the proximity of the lesion to surrounding anatomical structures. ${ }^{2}$ As PA radiographs are cost-effective and provide high-resolution imaging, they are still the most preferred imaging modalities. However, due to drawbacks, such as superimposition, distortion, and compression of anatomy of the area being radiographed, it is difficult to detect PA lesions. So, PA radiographs cannot consistently detect the site and the nature of a PA lesion. ${ }^{3-7}$

The ability to visualize the dentoalveolar structures in three dimensions has led cone-beam computed tomography (CBCT) to become an integral part of endodontics. The American Association of Endodontists (AAE) and the American Academy of Oral and Maxillofacial Radiology (AAOMR) guidelines recommend CBCT for the detection of pathosis, the assessment of previously treated teeth, the detection of vertical root fracture, the detection of traumatic injuries, the localization of resorptive defects, the presurgical treatment planning to locate root apex, the evaluation of proximity of the root apex and the lesion to anatomical structures, and the evaluation of healing following non-surgical and surgical endodontic treatment. ${ }^{8}$

Multiple studies have established the improved reliability in the diagnosis of PA lesions by CBCT over PA radiographs. ${ }^{9-11}$
${ }^{1-3}$ Department of Conservative Dentistry and Endodontics, Manubhai Patel Dental College and Hospital, Vadodara, Gujarat, India

Corresponding Author: Sonali Kapoor, Department of Conservative Dentistry and Endodontics, Manubhai Patel Dental College and Hospital, Vadodara, Gujarat, India, Phone: +91 9825323057, e-mail: docsonali@yahoo.com

How to cite this article: Sheth K, Kapoor S, Daveshwar S, et al. Comparison of Cone-beam Computed Tomography and Periapical Radiography to Determine the Proximity of Periapical Lesions to Anatomical Structures in Premaxillary Area prior to Surgical Endodontics: A Clinical Study. Int J Clin Pediatr Dent 2020;13(4):322-326. Source of support: Nil

Conflict of interest: None

Velvart et al., ${ }^{6}$ Low et al., ${ }^{12}$ and Huumonen et al. ${ }^{13}$ concluded from their studies that computed tomography helped correlate the relation of PA lesions to mandibular canal and maxillary sinus floor prior to PA surgery and also provided information for the retreatment cases.

Relationship of PA lesions in the maxillary anterior region to adjacent anatomical structures has not been evaluated yet. Knowledge of relation of PA lesion to the root apices, the nasopalatine canal, and the floor of nasal cavity can help in preventing complications, such as hypesthesia of anterior teeth and hard palate and damage to the nasal floor. Knowledge of proximity of the root apex to anterior nasal spine can help in deciding an alternative flap approach. Bone thickness and angulation of roots 
are important to determine the osseous entry and the amount of bone removal required to reach the root apex and PA lesion. ${ }^{14}$ Thus, the present study aimed to compare $C B C T$ and $\mathrm{PA}$ radiographs to determine the proximity of PA lesion to anatomical structures in the premaxillary area for decision-making before PA surgery.

\section{Materials and Methods}

Institutional review board approval was obtained. Twenty patients reporting to the Department of Conservative Dentistry and Endodontics were considered for the study. The study group comprised of 14 male (70\%) and 6 female (30\%) patients, with a mean age of $23.9 \pm 6.32$ years. History of present illness of all the patients was taken and they were examined clinically. After initial PA radiographs revealed the presence of PA lesion which required surgical intervention, they were considered for the study. Teeth included in the study were: single-rooted maxillary anterior teeth, teeth with previous root canal treatment, and teeth with previous restorations. Teeth that needed immediate therapy, teeth with radiographic evidence of fracture, unrestorable teeth, and pregnant women were excluded from the study.

\section{Image Acquisition}

\section{PA Radiographs}

A paralleling axis technique was used to obtain the PA radiographs with Biodent-1070-D (BioMedicare Imaging Co., Badlapur, Maharashtra, India) machine. A round collimator of 2.89" diameter with variable $\mathrm{kVp}$ and $\mathrm{mAs}$ and focal spot size $0.7 \times 0.7 \mathrm{~mm}$ was used. Images were captured using Sopix 2 sensors (Acteon, France). Images were presented using SOPRO imaging software (Acteon, France) as 8-bit Tagged Image File Format (TIFF). All images were compared and those which were found to be of adequate quality were selected and standardized for the study.

\section{CBCT}

The procedure was explained to the patient and consent was obtained before commencement of the scan. i-CAT ${ }^{\mathrm{TM}} \mathrm{Next}$ Generation CBCT scanner (Imaging Sciences International, Hatfield, Pennsylvania, USA) was used. Exposure factors used were $120 \mathrm{kVp}$, $5 \mathrm{~mA}$ with 7-second exposure time. Collimation suitable for the scan of maxilla was adjusted. The field of view (FOV) was $8 \times 5 \mathrm{~cm}$. The radiation dose varied according to the patients. Images were examined using the i-CAT software.

\section{Image Viewing}

Three endodontists with varying amounts of experience were selected. These observers determined proximity of PA lesions to anatomical structures for both PA radiographs and CBCT images (Figs 1 to 3 ) and selected treatment plan based on the two imaging modalities. All images were viewed on a HD LED Backlit Widescreen BrightView Display having a screen resolution of 1366 $\times 768$ pixels, brightness of $250 \mathrm{~cd} / \mathrm{m}^{2}{ }^{2}$ and contrast ratio 1000:1 installed with Microsoft Windows XP Service Pack 1 (Microsoft Corp, Redmond, Washington, USA). Patient information was anonymized and images were randomly presented. The observations were performed separately for PA radiographs and CBCT images. In the first evaluation, only the PA radiographs were presented. The same 20 cases with additional information of the CBCT scans were then reviewed by the examiners in the next evaluation. Evaluation for both the modalities was carried out after an interval of 10 days.

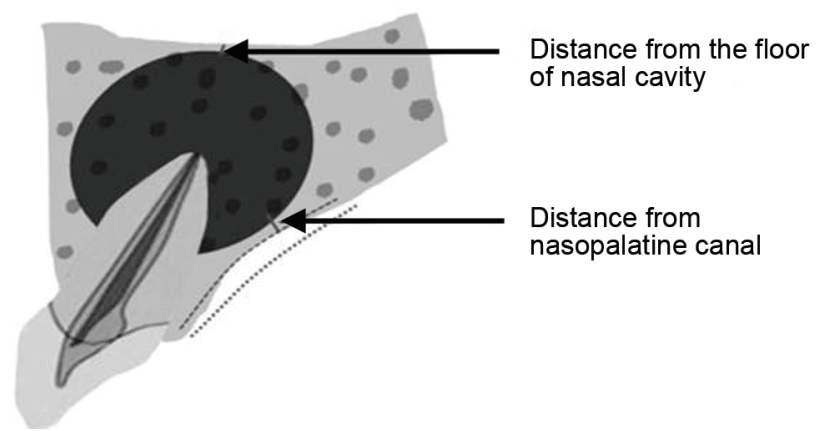

Fig. 1: Proximity of periapical lesion to anatomical structures in sagittal section

Each examiner was asked to analyze 10 randomly selected images again to check for consistency.

\section{Statistical Analysis}

The IBM SPSS (Version 17.0; SPSS Inc., Chicago, Illinois, USA) software was used for statistical analysis. To check for statistical relationship between the treatment plan given by PA radiographs and $\mathrm{CBCT}$ images, chi-square test was used. Reliability of the observations was evaluated using intraclass correlation (ICC). Interexaminer reliability was assessed by Cohen's kappa. Validity of the results was assessed by face validity.

\section{Results}

Distribution of study participants on the basis of anatomical structures involved and treatment plan is shown in Tables 1 and 2 , respectively. A significant difference in the treatment plan selected by observers between PA radiographs and CBCT images was revealed with the help of chi-square test $\left[\chi^{2}(3)=44.45\right.$, $p<0.05$ ]. Anatomical structures could be easily identified by all the observers. The ICC among observers regarding treatment plan and anatomical structures involved for both PA radiographs and $\mathrm{CBCT}$ images was calculated. Intraclass correlation for anatomical structures involved showed a high value $(0.958)$ which indicates a high similarity between the observations using PA radiographs and CBCT images (Table 3). Intraclass correlation for PA radiographs was 0.987 and for CBCT images was 0.968 which indicates a high similarity between the observations regarding the treatment plan using PA radiographs and CBCT images (Table 4). Measurement of agreement using PA radiographs was 0.844 and using CBCT images was 0.93 which indicates an almost perfect interexaminer reliability.

\section{Discussion}

The goal of PA surgery is to reinstate a tooth with an endodontic lesion by means of surgery. Periapical surgeries on maxillary anterior teeth generally involve fewer anatomical complications than posterior teeth. However, access to root apex in some patients may not be easy because of long roots, a shallow vestibule, or palatally inclined roots. The floor of nasal cavity can be encountered due to the presence of long roots or when PA lesion has extended superiorly and has eroded through the bony floor of the nose. The roots can also be close to bony anterior nasal spine which could complicate the route of entry to the root apex. Thus, for planning of PA surgery, extent of lesion, knowledge about the roots involved, relation between the lesion and adjacent anatomic 


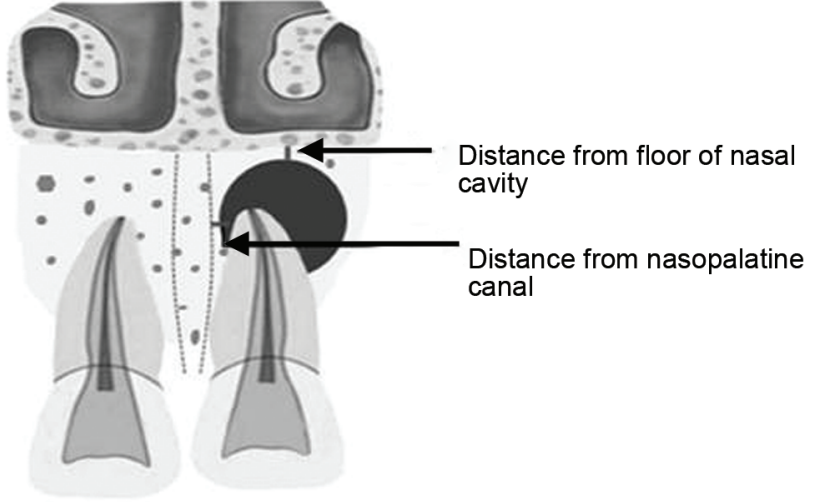

Fig. 2: Proximity of periapical lesion to anatomical structures in coronal section structures and between root apices need to be known to prevent any complications.

In the present study, three endodontists viewed images of both modalities and determined the proximity of PA lesions to anatomical structures and made treatment decisions according to each modality.

In case of PA radiographs, anatomical structures were either not visible or only the intermaxillary suture was seen. However, with the help of $C B C T$, proximity of lesions to anatomical structures, such as nasopalatine canal, anterior nasal spine, and nasal floor, could also be assessed. In the present study, with the help of CBCT, $50 \%$ of the lesions were found to be close to nasal floor leading to discontinuity or thinning of the nasal floor. $43.3 \%$ of lesions were found to be away from nasopalatine canal, while $36.7 \%$ of lesions were found to be in contact with the canal and $3.3 \%$ of the lesions were found to be involving the canal. These findings could not be
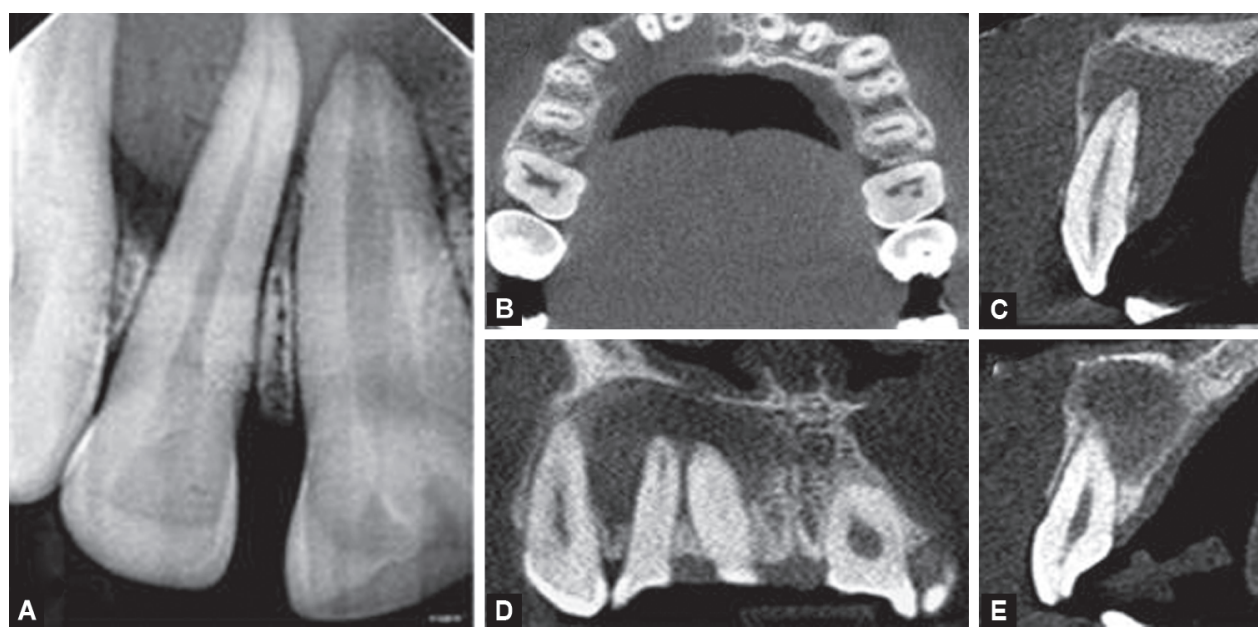

Figs 3A to E: (A) Periapical radiograph of maxillary central and lateral incisor with large periapical lesion; (B) Axial section of CBCT showing the buccopalatal extent of the lesion and proximity to nasopalatine canal; (D) Coronal section of CBCT showing the mesiodistal extent of the lesion; ( $C$ and E) Sagittal section of CBCT showing the buccopalatal extent of the lesion and involvement of palatal cortical plate

Table 1: Distribution of study participants according to anatomical structures involved

\begin{tabular}{|c|c|c|c|c|c|c|}
\hline \multirow[b]{3}{*}{ Anatomical structures involved } & \multicolumn{6}{|c|}{ Number of study participants } \\
\hline & \multicolumn{2}{|c|}{ Observer 1} & \multicolumn{2}{|c|}{ Observer 2} & \multicolumn{2}{|c|}{ Observer 3} \\
\hline & $R V G$ & $C B C T$ & RVG & $C B C T$ & $R V G$ & $C B C T$ \\
\hline Not visible & 22 & 0 & 22 & 0 & 23 & 0 \\
\hline Intermaxillary suture & 8 & 30 & 8 & 30 & 7 & 30 \\
\hline Nasopalatine canal & 0 & 30 & 0 & 30 & 0 & 30 \\
\hline Anterior nasal spine & 0 & 30 & 0 & 30 & 0 & 30 \\
\hline Nasal aperture & 0 & 30 & 0 & 30 & 0 & 30 \\
\hline
\end{tabular}

(RVG, radiovisiography; CBCT, cone-beam computed tomography)

Table 2: Distribution of study participants according to treatment plan

\begin{tabular}{|c|c|c|c|c|c|c|}
\hline \multirow[b]{3}{*}{ Treatment plan } & \multicolumn{6}{|c|}{ Number of study participants } \\
\hline & \multicolumn{2}{|c|}{ Observer 1} & \multicolumn{2}{|c|}{ Observer 2} & \multicolumn{2}{|c|}{ Observer 3} \\
\hline & RVG & $C B C T$ & $R V G$ & $C B C T$ & $R V G$ & $C B C T$ \\
\hline No endodontic treatment & 0 & 0 & 0 & 0 & 0 & 0 \\
\hline Root canal treatment & 3 & 0 & 3 & 0 & 3 & 0 \\
\hline Periapical surgery & 0 & 0 & 0 & 0 & 0 & 0 \\
\hline Apexification & 0 & 0 & 0 & 0 & 0 & 0 \\
\hline Re-root canal treatment & 3 & 0 & 3 & 0 & 2 & 0 \\
\hline Both root canal treatment and periapical surgery & 9 & 14 & 9 & 15 & 11 & 15 \\
\hline Root canal treatment, periapical surgery, and apexification & 15 & 16 & 15 & 15 & 14 & 15 \\
\hline
\end{tabular}


Treatment Planning: CBCT vs Periapical Radiograph

Table 3: Intraclass correlation coefficient for anatomical structures involved

\begin{tabular}{|c|c|c|c|c|c|c|c|}
\hline \multirow[b]{2}{*}{ Modality } & \multirow[b]{2}{*}{ Average measures } & \multicolumn{2}{|c|}{ 95\% Confidence interval } & \multicolumn{4}{|c|}{ F test with true value 0} \\
\hline & & Lower bound & Upper bound & Value & $d f 1$ & $d f 2$ & Sig. \\
\hline Periapical radiograph & 0.958 & 0.898 & 0.986 & 24.077 & 13 & 26 & 0.000 \\
\hline $\mathrm{CBCT}$ & 0.958 & 0.898 & 0.986 & 24.077 & 13 & 26 & 0.000 \\
\hline
\end{tabular}

Table 4: Intraclass correlation coefficient for treatment plan

\begin{tabular}{llllllll}
\hline & & \multicolumn{3}{c}{ 95\% Confidence interval } & \multicolumn{3}{c}{ F test with true value 0 } \\
\cline { 3 - 7 } Modality & Average measures & Lowerbound & Upper bound & Value & $d f 1$ & df2 & Sig. \\
\hline Periapical radiograph & 0.987 & 0.967 & 0.995 & 74.143 & 13 & 26 & 0.000 \\
CBCT & 0.968 & 0.922 & 0.989 & 31.462 & 13 & 26 & 0.000 \\
\hline
\end{tabular}

seen with the help of PA radiographs. This can be attributed to the fact that PA radiographs can produce acceptable images in only mesiodistal direction but information acquired in superio-inferior direction is limited thus, obscuring the visualization of anatomical structures that are located more superiorly. A similar problem in the detection of PA lesion and anatomical landmarks with PA radiographs was described by Velvart et al. ${ }^{6}$ Thus, the finding that PA radiographs can lead to miscalculation of the dimension of the PA lesion holds true. ${ }^{15-18}$

In case of treatment plan, there was a statistically significant difference $(p<0.05)$ between treatment plan given on the basis of $\mathrm{PA}$ radiographs and $\mathrm{CBCT}$ images. A change in examiner's treatment plan was recorded in $16 \%$ of the cases after viewing the CBCT scans. Similar observations were made in other studies which confirmed that preoperative CBCT imaging provides additional information which may lead to treatment plan modifications. ${ }^{19-21}$ The results of present study contradict those obtained by Balasundaram et al. ${ }^{22}$ who found that CBCT examination does not provide information that can change a therapy plan.

In the present study, CBCT images also provided additional important information which was not observed on PA radiographs. After the lesions were observed with $\mathrm{CBCT}$, there was an increase by $40 \%$ in the number of teeth found to be involved with the lesion. In a similar study, Lofthag-Hansen et al. ${ }^{9}$ could gather more information with the help of CBCT.

Low et al. ${ }^{12}$ and Patel et al. ${ }^{23}$ concluded in their respective studies that $\mathrm{CBCT}$ helped in the identification of lesions which were undetected on PA radiographs and this is possible as $C B C T$ removes the anatomical noise and irradiation geometry which hamper the PA radiographs. Thus, the observation that detection of lesions limited to the cancellous bone by PA radiographs can be difficult hold true. ${ }^{24-26}$

In the present study, $\mathrm{CBCT}$ also provided the advantage of helping in diagnosis of additional pathologies like the presence of external root resorption which was not observed on the PA radiographs. This finding was similar to the one obtained by Patel et al. ${ }^{27}$ who found that overall sensitivity of intraoral radiography in detecting external root resorption was lower than CBCT which in turn influenced the treatment decision.

During PA surgery, additional information like the thickness and shape of the bone and angulation of the roots can be useful in determining the osseous entry. ${ }^{6}$ These findings could be obtained with the help of CBCT by selecting relevant views. In the present study, thinning of both the buccal and palatal cortical plates was found in around $40 \%$ of the cases. This information can be beneficial in determining the amount of bone needed to be removed to gain access to the PA lesion and the root apex. ${ }^{6}$ In a study Nakata et al. ${ }^{28}$ concluded that by selecting relevant views and slices of data of $C B C T$, thickness of cortical plate, pattern of cancellous bone, and angulation of roots of teeth could be viewed. Yoshioka et al. ${ }^{29}$ concluded that in cases with persistent PA lesions, CBCT helped in correct identification of the type of PA bone defect. Rigolone et al. ${ }^{30}$ found that CBCT helped in measuring the distance between cortical plates and palatal root of maxillary first molar. Velvart et al. ${ }^{6}$ demonstrated that CT scans could accurately determine the thickness of bone and the extent of PA lesion.

In the present study, with regards to the number of tooth surfaces involved, there was change in the findings after lesions were assessed with $C B C T$ as it facilitated a complete threedimensional viewing of the tooth involved with the lesion.

These findings highlight the fact that preoperative $\mathrm{CBCT}$ images are able to provide more diagnostically relevant information as compared to PA radiographs. In case of treatment planning for PA surgery, $C B C T$ provided clinically relevant additional information which could not be obtained from PA radiographs.

\section{Conclusion}

The present study showed that $\mathrm{CBCT}$ images revealed an increase in the number of teeth involved with the lesion. A change in the treatment plan was observed after viewing additional information with $\mathrm{CBCT}$. Thus, $\mathrm{CBCT}$ helps in accurate treatment planning and providing safer treatment by presenting the clinician with relevant information, such as thickness of bone, angulation of the root, and position of anatomical structures.

\section{Clinical Significance}

- High standard radiographs play an important role in preoperative diagnosis and treatment planning of teeth scheduled for PA surgery.

- In case of maxillary anterior teeth, knowledge of relation of root apices to lesion, nasopalatine canal, and floor of nasal cavity will help in preventing complications to anterior teeth and prevent damage to hard palate and nasal floor.

- Knowledge of proximity of root apex to anterior nasal spine will help in deciding an alternative osseous approach.

- Bone thickness and angulation of roots help to ascertain the osseous entry and amount of bone removal required to reach the root apex and PA lesion. 
- Comparison between the findings obtained through PA radiographs and $C B C T$ images revealed that $C B C T$ provided a three-dimensional view which gave additional information which helped in deciding the flap approach and also helped in determining if any additional treatment was required.

\section{References}

1. Marx RE, Stern D. Oral and maxillofacial pathology: a rationale for diagnosis and treatment. Illinois: Quintessence Int 2003. 574-579.

2. Reit $C$, Petersson K. Diagnosis of pulpal and periradicular disease Bergenholtz G, Horsted-Bindslev P, Reit C, ed. Textbook of Endodontology. 2nd ed., UK: Wiley-Blackwell; 2010. pp. 235-253.

3. Kundel HL, Revesz G. Lesion conspicuity, structured noise, and film reader error. Am J Roentgenol 1976;126(6):1233-1238. DOI: 10.2214/ ajr.126.6.1233.

4. Garcia de Paula-Silva FW, Hassan B, Bezerra da Silva LA, et al. Outcome of root canal treatment in dogs determined by Periapical radiography and cone-beam computed tomography scans. J Endod 2009;35(5):723-726. DOI: 10.1016/j.joen.2009.01.023.

5. Van Vorde HE, Bjorndahl AM. Estimated endodontic working length with paralleling radiographs. Oral Surg Oral Med Oral Pathol Oral Radiol Endod 1969;27(1):106-110. DOI: 10.1016/0030-4220(69) 90037-1.

6. Velvart $P$, Hecker $H$, Tillinger $G$. Detection of the apical lesion and the mandibular canal in conventional radiography and computed tomography. Oral Surg Oral Med Oral Pathol Oral Radiol Endod 2001;92(6):682-688. DOI: 10.1067/moe.2001.118904.

7. Forsberg J, Halse A. Radiographic simulation of a periapical lesion comparing the paralleling and the bisecting- angle techniques. Int Endod J 1994;27(3):133-138. DOI: 10.1111/j.1365-2591.1994. tb00242.x.

8. $A A E$ and $A A O M R$ Joint Position Statement. Use of cone beam computed tomography in endodontics 2015 update. Oral Surg Oral Med Oral Pathol Oral Radiol 2015;120(4):508-512. DOI: 10.1016/j. oooo.2015.07.033.

9. Lofthag-Hansen S, Huumonen S, Gröndahl K, et al. Limited conebeam CT and intraoral radiography for the diagnosis of periapical pathology. Oral Surg Oral Med Oral Pathol Oral Radiol Endod 2007;103(1):114-119. DOI: 10.1016/j.tripleo.2006.01.001.

10. Bornstein $M$, Lauber $R$, Sendi $P$, et al. Comparison of periapical radiography and limited cone-beam computed tomography in mandibular molars for analysis of anatomical landmarks before apical surgery. J Endod 2011;37(2):151-157. DOI: 10.1016/j.joen.2010.11.014.

11. Estrela C, Bueno MR, Leles CR, et al. Accuracy of cone beam computed tomography and panoramic radiography for the detection of apical periodontitis. J Endod 2008;34(3):273-279. DOI: 10.1016/j. joen.2007.11.023.

12. Low KM, Dula K, Bürgin W, et al. Comparison of periapical radiography and limited cone-beam tomography in posterior maxillary teeth referred for apical surgery. J Endod 2008;34(5):557-562. DOI: 10.1016/j. joen.2008.02.022.

13. Huumonen S, Kvist T, Grondahl K, et al. Diagnostic value of computed tomography in retreatment of root fillings in maxillary molars. Int Endod J 2006;39(10):827-833. DOI: 10.1111/j.1365-2591. 2006.01157.x.

14. Bigras BR, Johnson BR, BeGole EA, et al. Differences in clinical decision making: a comparison between specialist and general dentists. Oral Surg Oral Med Oral Pathol Oral Radiol Endod 2008;106(1):139-144. DOI: 10.1016/j.tripleo.2008.01.037.
15. Bender IB, Selzer S. Roentgenographic and direct observation of experimental lesions in bone: I. J Am Dent Assoc 1961;62(2):152-160. DOI: 10.14219/jada.archive.1961.0030.

16. Schwartz SF, Foster JK. Roentgenographic interpretation of experimentally produced bone lesions. Oral Surg Oral Med Oral Pathol Oral Radiol Endod 1971;32(4):606-612. DOI: 10.1016/00304220(71)90326-4.

17. Lee S-J, Messer HH. Radiographic appearance of artificially prepared periapical lesions confined to cancellous bone. Int Endod J 1986;19(2):64-72. DOI: 10.1111/j.1365-2591.1986.tb00894.x.

18. Wallace JA, Nair MK, Colaco MF, et al. A comparative evaluation of the diagnostic efficacy of film and digital sensors for detection of simulated periapical lesions. Oral Surg Oral Med Oral Pathol Oral Radiol Endod 2001;92(1):93-97. DOI: 10.1067/moe.2001.115974.

19. Ee J, Fayad MI, Johnson BR. Comparison of endodontic diagnosis and treatment planning decisions using cone beam volumetric tomography versus periapical radiography. J Endod 2014;40(7):910916. DOI: 10.1016/j.joen.2014.03.002.

20. Mota de Almeida FJ, Knutsson K, Flygare L. The effect of cone beam CT (CBCT) on therapeutic decision-making in endodontics. Dentomaxillofac Radiol 2014;43(4):20130137. DOI: 10.1259/ dmfr.20130137.

21. Rodriguez G, Abella F, Durán-Sindreu F, et al. Influence of cone-beam computed tomography in clinical decision making among specialists. J Endod 2017;43(2):194-199. DOI: 10.1016/j.joen.2016.10.012.

22. Balasundaram A, Shah P, Hoen MM, et al. Comparison of cone-beam computed tomography and periapical radiography in predicting treatment decision for periapical lesions: a clinical study. Int J Dent 2012;2012:1-8. DOI: 10.1155/2012/920815.

23. Patel S, Dawood A, Mannocci F, et al. Detection of periapical bone defects in human jaws using cone beam computed tomography and intraoral radiography. Int Endod J 2008;42(6):507-515. DOI: 10.1111/j.1365-2591.2008.01538.x.

24. Shoha RR, Dowson J, Richards AG. Radiographic interpretation of experimentally produced bone lesions. Oral Surg Oral Med Oral Pathol Oral Radiol Endod 1974;38(2):294-303. DOI: 10.1016/00304220(74)90071-1.

25. Scarfe WC, Czerniejewski VJ, Farman AG, et al. In vivo accuracy and reliability of color-coded image enhancements for the assessment of periradicular lesion dimensions. Oral Surg Oral Med Oral Pathol Oral Radiol Endod 1999;88(5):603-611. DOI: 10.1016/s1079-2104(99)70093-4.

26. Marmary $Y$, Koter $T$, Heling I. The effect of periapical rarefying osteitis on cortical and cancellous bone. A study comparing conventional radiographs with computed tomography. Dentomaxillofac Radiol 1999;28(5):267-271. DOI: 10.1038/sj/dmfr/4600453.

27. Patel $S$, Dawood A, Wilson R, et al. The detection and management of root resorption lesions using intraoral radiography and cone beam computed tomography - an in vivo investigation. Int Endod J 2009;42(9):831-838. DOI: 10.1111/j.1365-2591.2009.01592.x.

28. Nakata K, Naitoh M, lzumi M, et al. Effectiveness of dental computed tomography in diagnostic imaging of periradicular lesion of each root of a multirooted tooth: a case report. J Endod 2006;32(6):583-587. DOI: 10.1016/j.joen.2005.09.004.

29. Yoshioka T, Kikuchi I, Adorno CG, et al. Periapical bone defects of root filled teeth with persistent lesions evaluated by cone-beam computed tomography. Int Endod J 2011;44(3):245-252. DOI: 10.1111/j.1365-2591.2010.01814.x.

30. Rigolone $M$, Pasqualini $D$, Bianchi $L$, et al. Vestibular surgical access to the palatine root of the superior first molar: "low-dose cone-beam" CT analysis of the pathway and its anatomic variations. J Endod 2003;29(11):773-775. DOI: 10.1097/00004770-200311000-00021. 